\title{
Recent Status and Future Prospects of Coaxial-Cavity Gyrotron Development at KIT
}

\author{
Stefan Illy ${ }^{1}$, Konstantinos A. Avramidis ${ }^{1}$, Gerd Gantenbein ${ }^{1}$, Zisis Ioannidis ${ }^{1}$, Jianbo Jin ${ }^{1}$, Parth C. Kalaria ${ }^{1}$, \\ Ioannis Gr. Pagonakis ${ }^{1}$, Sebastian Ruess ${ }^{1,2}$, Tobias Ruess ${ }^{1}$, Tomasz Rzesnicki ${ }^{1}$, Manfred Thumm $^{1,2}$, and John Jelonnek ${ }^{1,2}$ \\ ${ }^{1}$ IHM, Karlsruhe Institute of Technology (KIT), Kaiserstr. 12, 76131 Karlsruhe, Germany \\ ${ }^{2} I H E$, Karlsruhe Institute of Technology (KIT), Kaiserstr. 12, 76131 Karlsruhe, Germany
}

\begin{abstract}
Coaxial-cavity gyrotrons are microwave sources that can extend the possible power levels of hollow cavity gyrotrons significantly and make them attractive for future fusion experiments and power plants. KIT already demonstrated operation of a modular short-pulse $170 \mathrm{GHz}$ coaxial-cavity gyrotron with an output power of $2.2 \mathrm{MW}$, operating in the $\mathrm{TE}_{34,19}$ mode. Today's focus of KIT is the verification of this technology at longer pulses, which will prove the long-pulse capabilities also. At the moment, the current KIT prototype has been extended with cooling capabilities for all critical, highly loaded components of the tube and two new, different electron guns are available for operation. This paper will give an overview about the recent status and the future prospects connected to the coaxial-cavity gyrotron development at KIT, including both experimental and theoretical activities.
\end{abstract}

\section{Introduction}

At KIT, the activities in gyrotron research and development shall lead to a gyrotron design that will fulfil the increasing requirements for future nuclear fusion experiments and power plants, like DEMO [1], the fusion demonstration power plant that will follow ITER. Today, the requirements for the initial gyrotron installation at ITER are an RF output power capability of $1 \mathrm{MW}(3600 \mathrm{~s})$ at an operating frequency of $170 \mathrm{GHz}$ for each single gyrotron [2]. For a future DEMO an RF output power level of minimum $2 \mathrm{MW} \mathrm{CW}$ at even higher operating frequencies up to $240 \mathrm{GHz}$ is under consideration $[3,4,5]$. It is expected that the coaxialcavity gyrotron $[6,7]$ technology will allow an operation at those output power levels. Compared to the conventional hollow-cavity gyrotron technology as used for e.g. Wendelstein7-X (W7-X) and ITER today, the coaxial-cavity gyrotron technology allows the effective reduction of the voltage depression due to the space charge effect of the electron beam and the reduction of the mode competition due to the mode selectivity of the carefully chosen longitudinally corrugated inner conductor at high operating frequencies. The key challenges in the construction and operation of a coaxialcavity gyrotron are the design of the coaxial insert in terms of beam-wave interaction, appropriate cooling of the insert and an accurate alignment of this relatively long structure. First experimental results obtained with the $170 \mathrm{GHz} 2 \mathrm{MW}$ short-pulse coaxial-cavity preprototype gyrotron (Fig. 1) at pulse length of a few milliseconds (ms) have shown the potential of the coaxial-cavity concept in the multi-MW operation regime. Nonetheless, until today, the coaxial-cavity gyrotron technology has not been verified at longer pulses above a few ms. Therefore, a focus of KIT is the verification of this technology at pulse lengths up to one second which will prove the long-pulse capabilities for longer pulses also. For the very first time, a coaxial cavity gyrotron will be operated and investigated in the experiment in long pulse operation.

At the moment, the current KIT prototype has been extended with cooling capabilities for all critical, highly loaded components of the tube [8]; a new triode electron gun equipped with non-emissive coating at the emitter edges [9] has been procured and a new inverse triode gun has been manufactured in-house [10]; both electron guns should increase the electron beam quality and therefore the overall performance and operation stability of the tube.

Intense theoretical design activities to support the manufacturing have been performed and are still ongoing to assist during the experimental activities. This includes the design and optimization of different kinds of Magnetron Injection Guns (MIGs) [11], the simulation of the gyrotron interaction [12,13] and quasi-optical output launcher [14,15] (focusing also on a future dual frequency operation at $170 \mathrm{GHz} / 204 \mathrm{GHz}$ ). In addition, this also includes multi-physics simulations of advanced cooling concepts for the outer cavity wall of the resonator [16] (including the manufacturing of mockups), fluid-dynamics simulations of the cooling structure of the coaxial insert [17] and investigations of enhanced longitudinal sweeping concepts for the collector $[18,19]$.

Corresponding author: stefan.illy@kit.edu 
This paper is organized as follows: In Section 2, the current status and recent major results of the coaxialcavity gyrotron development at KIT are presented; in Section 3, the design and manufacturing of the key components for the extension to longer pulses are described. Section 4 then presents the planned experimental activities in the near future.

\section{Current Experimental Status}

During the experimental short-pulse test campaign, the already existing KIT $170 \mathrm{GHz} 2 \mathrm{MW}$ coaxial-cavity modular pre-prototype did show an excellent and very stable performance. The tube was operated at an acceleration voltage of up to $\mathrm{U}_{\mathrm{c}}=93 \mathrm{kV}$ and an electron beam current of $\mathrm{I}_{\mathrm{b}}=80 \mathrm{~A}$. At the nominal operating parameters, listed in Table $1\left(\mathrm{U}_{\mathrm{c}}=90 \mathrm{kV}, \mathrm{I}_{\mathrm{b}}=75 \mathrm{~A}\right)$, an RF output power of $2 \mathrm{MW}$ up to $10 \mathrm{~ms}$ pulse length has been achieved. An electronic (interaction) efficiency between the electron beam and the electromagnetic field in the cavity of $\sim 30 \%$ was achieved at nominal operating parameters [20]. During the experiments the magnetic field of the superconducting magnet was set to $6.90 \mathrm{~T}$. The acceleration voltage was raised up to $\sim 93 \mathrm{kV}$. At the same time, the electron beam current was set to $80 \mathrm{~A}$. That allowed to generate a world-record in RF output power of $2.2 \mathrm{MW}$ at $170 \mathrm{GHz}$ with an efficiency close to $30 \%$. The calorimetrically measured RF output power and electronic efficiency versus cathode voltage is shown in Fig. 2. The achieved results are in excellent agreement with the multi-mode simulations performed at KIT [20].

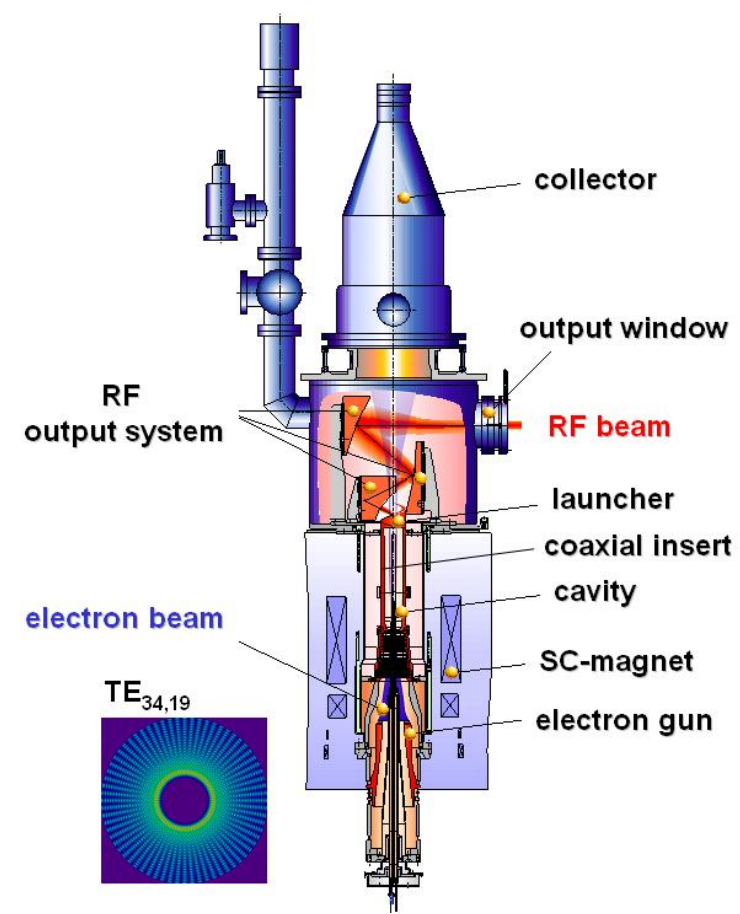

Fig. 1. Schematic sketch of the KIT $2 \mathrm{MW}, 170 \mathrm{GHz}$ shortpulse coaxial cavity gyrotron.
The experimental results were performed with an improved design of the launcher [14] with smoothed inner wall perturbations. That launcher is the major part of the quasi-optical system which converts the very highorder cavity mode into a Gaussian beam. The efficiency of the mode converter had been verified in low power measurements before implementing that launcher into the pre-prototype. The measured results are in excellent agreement with the expectations from numerical analysis. The fundamental Gaussian mode content had been extracted from the measured patterns and was calculated to be around $96 \%$, which corresponds very well to the simulated value of $96.3 \%$. In addition, at high output power, the profile of the RF beam had been measured with an infrared camera (IR-camera) (Fig. 3 [20]) at several different distances from the output window. The analysis of the data verified again the very good quality of the generated RF beam and it is in accordance with the low power measurements. The amount of the RF stray radiation losses inside the gyrotron tube had been obtained by measuring the RF power radiated through the relief window with a very sensitive ballistic calorimeter. Finally the stray radiation losses with the new launcher are significantly reduced from $7 \%$ (previous experiments) down to $4 \%$ [20].

Table 1. Design parameters for the coaxial-cavity short pulse gyrotron.

\begin{tabular}{|l|r|}
\hline Operating cavity mode & $\mathrm{TE}_{34,19}$ \\
\hline Frequency $(\mathrm{f})$ & $170 \mathrm{GHz}$ \\
\hline RF output power $\left(\mathrm{P}_{\text {out }}\right)$ & $2 \mathrm{MW}$ \\
\hline Beam current $\left(\mathrm{I}_{\mathrm{b}}\right)$ & $75 \mathrm{~A}$ \\
\hline Accelerating voltage $\left(\mathrm{U}_{\mathrm{c}}\right)$ & $90 \mathrm{kV}$ \\
\hline Velocity ratio (pitch factor) $(\alpha)$ & $\sim 1.3$ \\
\hline Electron beam radius & $10.0 \mathrm{~mm}$ \\
\hline Cavity magnetic field $\left(\mathrm{B}_{\mathrm{cav}}\right)$ & $6.87 \mathrm{~T}$ \\
\hline $\begin{array}{l}\text { Overall efficiency }(\text { with } \\
\text { depressed collector) }\end{array}$ & $50 \%$ \\
\hline $\begin{array}{l}\text { Pulse length (operation phase } 1) \\
\text { Pulse length (operation phase } 2)\end{array}$ & $150 \mathrm{~ms}$ \\
&
\end{tabular}

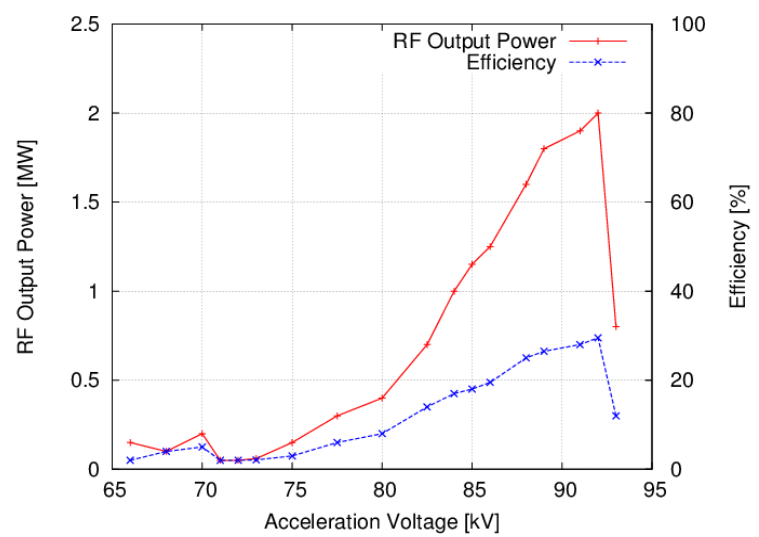

Fig. 2. Measured RF output power and overall efficiency as a function of the accelerating voltage $U_{c}$ (obtained at $B_{\text {cav }}=6.87 \mathrm{~T}$ and $I_{b} \sim 75 \mathrm{~A}$, operation without depressed collector). 


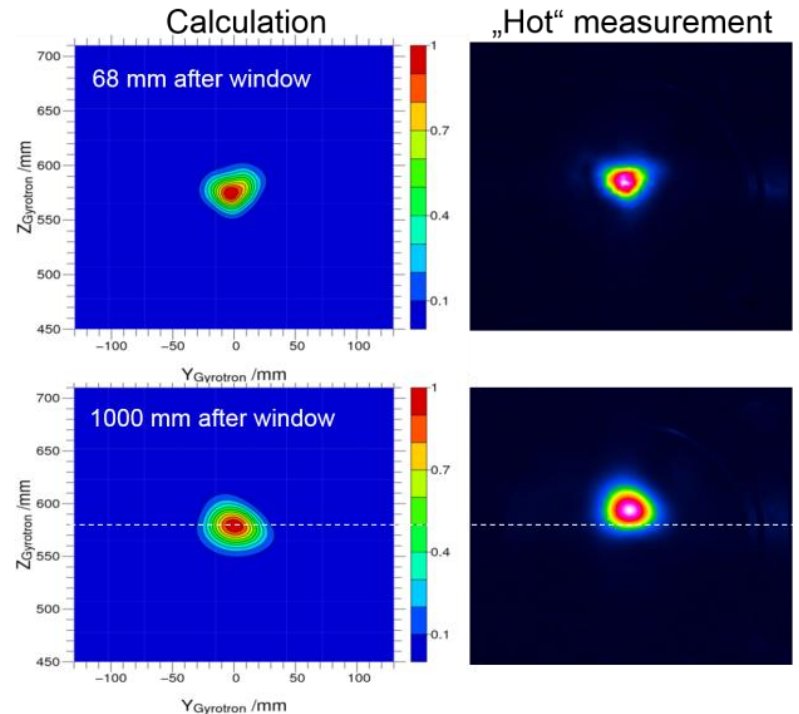

Fig. 3. Intensity distribution of the calculated and measured (IR-image) gyrotron RF output beam in $68 \mathrm{~mm}$ and $1000 \mathrm{~mm}$ distance from the window [21] (qualitative comparison).
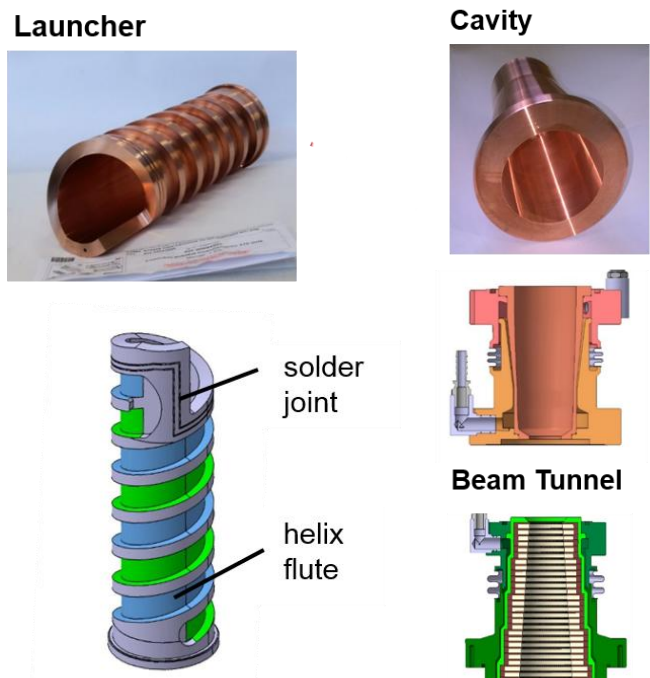

Beam Tunnel

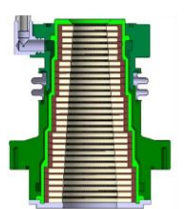

Fig. 4. Water-cooled subcomponents of the KIT $170 \mathrm{GHz}$ 2 MW coaxial cavity gyrotron for pulse lengths up to $1 \mathrm{~s}$.

\section{Design and Manufacturing of the Coaxial-Cavity Longer Pulse Pre- Prototype Gyrotron}

In order to increase the pulse length from the ms-range up to the range of one second, the main components of the $2 \mathrm{MW}$ coaxial-cavity gyrotron such as the beam tunnel, the cavity, the launcher, the mirrors, the chemical vapor deposited (CVD) diamond RF output window and the collector have to be equipped with an active cooling system. The corresponding design activity was starting in 2015. The already manufactured main components of the tube are shown in Fig. 4. One of the main project requirements is to keep the modularity of the gyrotron. Therefore, an independent cooling system for each component is considered. This allows the monitoring of the internal losses in each gyrotron component and of the final energy balance of the tube during longer-pulse operation. In the following, the advanced key components of the gyrotron are described.

\subsection{Inverse Magnetron Injection Gun}

A major step towards higher output power and operating frequency is the Inverse Magnetron Injection Gun (IMIG) [10]. An IMIG has been designed and already manufactured for the KIT 2 MW coaxial-cavity gyrotron [22]. It is expected that this gun leads to a more stable operation at even higher output power due to the avoidance of potential wells and secondary electrons. Due to the triode configuration of the IMIG, including a separate modulation anode, the IMIG has the flexibility to be operated at multiple frequencies such as $170 \mathrm{GHz}$ and $204 \mathrm{GHz}$. Because of the possibility of larger emitter radius the gyrotron can be operated at a larger operating beam current and therefore at a higher RF output power. The requested electron beam parameters are presented in Table 1 together with the operation parameters of the tube. The simulated parameters of the IMIG indicate a high electron beam quality [10], which promises a reliable gyrotron operation at nominal operating parameters. Additionally, the IMIG is the first MIG which fulfils the design criteria for preventing the generation of trapped electrons as presented in [23]. In the existing IMIG design the emitter radius is set to $62 \mathrm{~mm}$ with an emitter thickness of $5 \mathrm{~mm}$. The emitter has an angle of $25^{\circ}$ with respect to the $\mathrm{z}$-axis of the tube. In order to keep the temperature in the neighbouring regions of the emitter low at both edges, isolation gaps with a width of $200 \mu \mathrm{m}$ are designed. As a result, the electrons, emitted from the emitter edges have a very high pitch factor, which is related to the field enhancement at the emitter edges. In order to mitigate this effect, the emitter is pushed into the cathode by $70 \mu \mathrm{m}$ to reduce the local electric field at the edges.

In order to minimize mechanical shifts and misalignments caused by thermal effects, the thermomechanical behaviour of the IMIG was investigated by the use of COMSOL. In comparison to the "conventional" MIG (Fig. 6), the cathode and emitter of the IMIG (Fig. 5) are placed at the outside of the whole structure. Hence, it is directly cooled by passing oil at the outer surface of the cathode elements. That is the major benefit if comparing to the standard MIG used in all conventional gyrotrons. The components of the cathode and anode are made of materials with high thermal conductivity, mainly $\mathrm{CuCrZr}$ (indicated in Fig. 5 using orange colour) and Molybdenum (Fig. 5, marked by dark blue colour). Therefore, the heat loading of the neighbouring emitter regions is directly guided outside of the MIG and absorbed by the oil flow. Compared to conventional MIGs the temperature of the loaded parts is significantly reduced by $40 \%$ down to $\sim 150{ }^{\circ} \mathrm{C}$. Corresponding simulation results are presented in [24].

Another significant influence on the beam quality, hence on the generated output power and efficiency, is caused by an inhomogeneous temperature distribution at the surface of the emitter. Obviously an inhomogeneous 
temperature distribution leads to an inhomogeneous emission. The emission inhomogeneity can be experimentally investigated by measuring the CurrentVoltage Characteristics as published in [25]. Measurements of the temperature distribution of the emitter of the inverse MIG did indicate an azimuthal sinusoidal temperature distribution. The expected nominal temperature was $1000^{\circ} \mathrm{C}$ whereas the measured temperature varied between $993^{\circ} \mathrm{C}$ and $1007^{\circ} \mathrm{C}$. One explanation for the non-constant azimuthal temperature distribution is the fact that the ends of the heater filament are not overlapping each other.

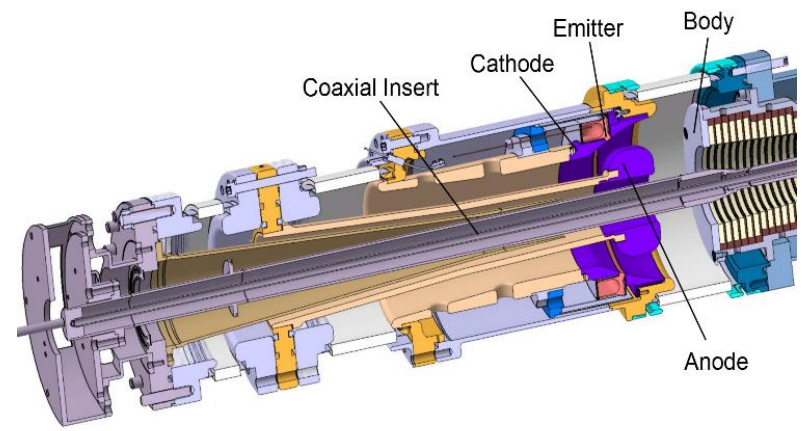

Fig. 5. Sketch of the already manufactured inverse magnetron injection gun (IMIG); the total length of the displayed section is approximately $700 \mathrm{~mm}$.

\subsection{Advanced Conventional Magnetron Injection Gun}

The enhancement of the electric field at the emitter edges has a significant influence regarding the electron beam quality. Tolerance studies of the radial emitter position have confirmed those observations and have shown that manufacturing tolerances of some microns already become critical. A possible solution for this major issue is the implementation of an anti-emission coating at the edges of the emitter. For example, considering a misalignment of $\pm 80 \mu \mathrm{m}$, the variation of the pitch factor measured over the emitter area is $83.3 \%$ smaller compared to the variation using a conventional emitter [23]. A new conventional MIG with an advanced coated emitter for the $170 \mathrm{GHz} 2 \mathrm{MW}$ longer-pulse coaxial-cavity gyrotron, as shown in Fig. 6, has been designed by KIT and manufactured by Thales Electron Device (France) and is now available for experiments [11].

\subsection{Beam Tunnel}

The beam tunnel consists of alternating stacked copper rings and rings of lossy ceramics. Compared to the other components of the gyrotron, the thermal loading is relatively low; however, an active cooling is also considered for this component (see Fig. 4), which will allow to estimate the losses in the ceramic discs caused by RF stray radiation and spurious oscillations in the beam tunnel, if present.

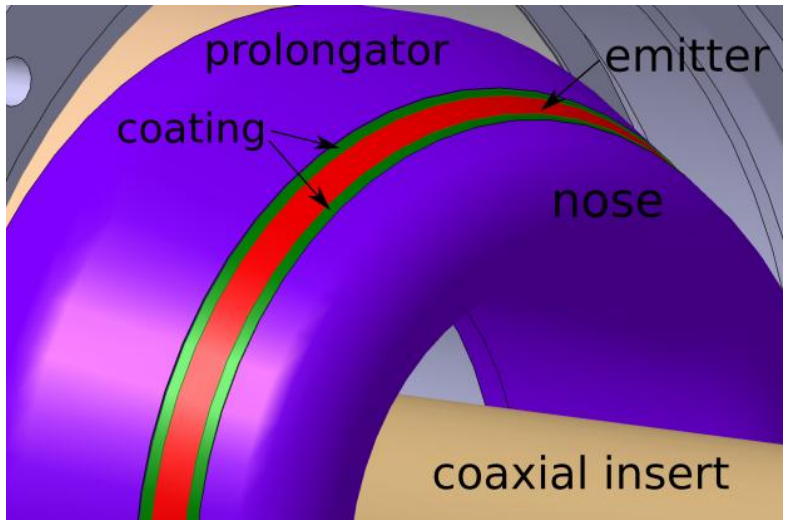

Fig. 6. Cathode of the advanced conventional magnetron injection gun (MIG) with an anti-emissive coating at the emitter edges (indicated in green).

\subsection{Cavity}

In the gyrotron cavity, the perpendicular kinetic energies of the individual electrons of the electron beam are transferred to the electromagnetic field. The expected total power loss in the cavity due to thermal wall loading is about $50 \mathrm{~kW}$ at nominal $2 \mathrm{MW}$ gyrotron operation. Due to the high quality factor of the cavity one finds the peak thermal wall loading of approximately $2 \mathrm{~kW} / \mathrm{cm}^{2}$ in a very narrow region at the center of the cavity. It leads to a very high temperature gradient at the inner side of the cavity wall, which results in thermal stress, and, finally leads to a deformation of the cavity wall. Depending on that wall deformation, the operating frequency of the gyrotron is shifted and the quality factor of the cavity is changing significantly. Therefore, it is mandatory to implement effective water cooling. The chosen approach of the active cooling system is similar to the design of the cooling system for the launcher. However, due to the position and orientation, the implementation of a helix-type channel system is not necessary. The different temperatures and material properties of the coat and the outer cavity wall require also the implementation of a bellow for compensation of thermal expansion. The reduction of the water cooling gap (see Fig. 4, cavity) increases the flow velocity of the water and therefore the cooling capacity. The limitation of the cavity is a maximum temperature of approximately $350{ }^{\circ} \mathrm{C}$. Based on this, the multi-physics software COMSOL predicts a maximum pulse length of approximately $1 \mathrm{~s}$.

Additionally, the cavity uptaper is extended and optimized. The simulation results predict a transmission of $99.94 \%$ of the $\mathrm{TE}_{34,19}$-mode and a very low conversion to unwanted spurious modes.

\subsection{Quasi-Optical Launcher}

As stated before, the launcher together with the mirror system is responsible for the conversion of the cavity $\mathrm{TE}_{34,19}$-mode into the fundamental Gaussian mode of the RF output beam [14]. Especially, at the tip of the launcher, where the complete RF-power is finally 
focused, the thermal loading of $0.4 \mathrm{~kW} / \mathrm{cm}^{2}$ is critical. In order to achieve a stable operation, it is necessary to cool that launcher tip in an efficient way. To achieve that, the water inlet and outlet of the launcher is connected at the bottom of the launcher. A helix cooling structure has been used to make that configuration possible. Due to that helix structure (see. Fig. 4) the channels have to be milled with a 5-axis milling cutter. In order to caulk the channels a stainless steel coat is imposed and brazed from the outside of the launcher. The introduction of a bellow is necessary as the launcher and the coat consist of different materials that have different thermal expansion coefficients. The already manufactured launcher shows a desired surface quality. The tolerances are in the range of below $\pm 10 \mu \mathrm{m}$. The launcher performance has been successfully verified in a cold measurement test setup. Fig. 7 shows the radiated pattern which was sampled at a distance of $10 \mathrm{~cm}$ from the axis to the position of the first mirror. The measurement results correspond well to the simulation results and the measurements on the previous launchers. The simulated radiation pattern has been calculated with the full wave 3 D vector analysis code SURF3D [26]. Technologically, soldered and brazed joints with a leakage rate of $<10^{-12}$ mbar $1 / \mathrm{s}$ were achieved for the launcher parts.
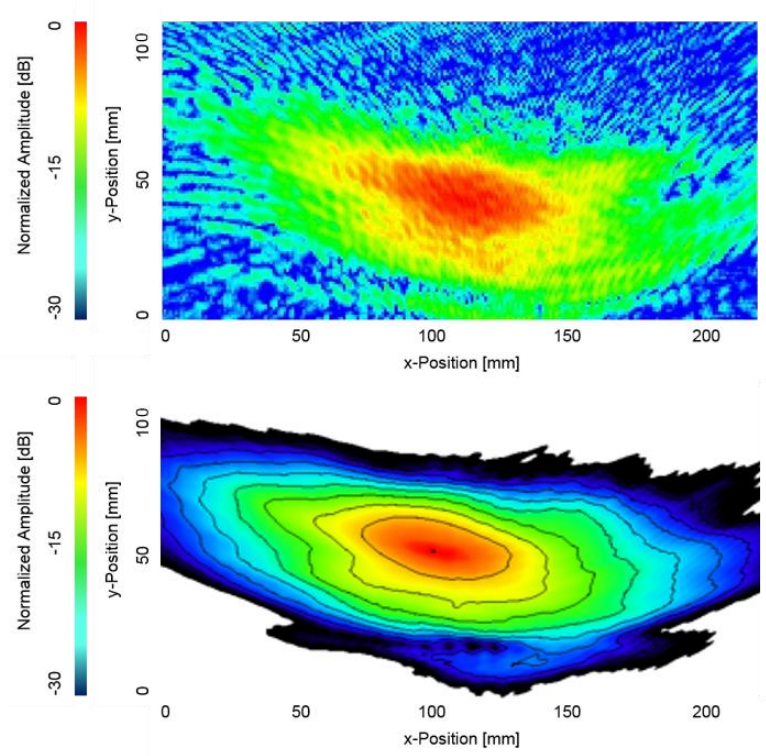

Fig. 7. Radiation pattern of the water-cooled launcher (top: cold measurement; bottom: corresponding simulation results).

\section{Experimental Activities in the Near Future}

During the last months, the assembly of the whole gyrotron has been finished and the tube has been equipped with a relatively old MIG for first verifications. The performed short-pulse measurements with pulse lengths $\leq 2.5 \mathrm{~ms}$ showed the same behaviour and output power levels as described in Section 2.

At the moment, the old MIG has been replaced by the new conventional triode type MIG with anti-emissive emitter edge coating (described in section 3.2) and new results will be expected during the next months. After finishing this measurement campaign, the experiments will be continued with the new IMIG.

\section{Conclusion}

KIT is pushing forward the coaxial-cavity gyrotron development by building up a modular longer-pulse 170 $\mathrm{GHz}, 2 \mathrm{MW}$ pre-prototype targeting at pulse lengths of up to $1 \mathrm{~s}$. The design principle and the manufacturing process of that pre-prototype have been presented here. All the subcomponents have been manufactured, brazed and successfully tested regarding vacuum tightness. Furthermore, the IMIG was manufactured at KIT with an expected high electron beam quality. Additionally, an advanced conventional MIG with coated emitter edges has been designed by KIT and manufactured at Thales Electron Devices. Both MIGs can be operated in hollow cavity as well as coaxial-cavity gyrotrons. The longerpulse $2 \mathrm{MW}$ coaxial-cavity gyrotron and the two MIGs will be tested during the next months.

Theoretical design activities are also on-going, focusing on possible two-frequency operation of the $2 \mathrm{MW}$ coaxial cavity gyrotron at $170 \mathrm{GHz}$ and $204 \mathrm{GHz}$ [13].

This work has been carried out within the framework of the EUROfusion Consortium and has received funding from the Euratom research and training programme 2014-2018 under grant agreement No 633053. The views and opinions expressed herein do not necessarily reflect those of the European Commission. Part of the simulations was performed on the EUROfusion High Performance Computer (Marconi-Fusion).

\section{References}

1. G. Federici et al., 13th International Symposium on Fusion Nuclear Technology, 25-29 September 2017, Kyoto, Japan, to appear in Fus. Eng. Des.

2. J. Jelonnek et al., IEEE Trans. Plasma Sci., 42, Issue 5, pp. 1135-1144. 2014, doi: 10.1109/TPS.2014.2301839.

3. J. Franck, Systematic Study of Key Components for a Coaxial-Cavity Gyrotron for DEMO, KIT Scientific Publishing, Karlsruhe, 2017.

4. J. Jelonnek et al., Fusion Engineering and Design, 2017, doi: 10.1016/j.fusengdes.2017.01.047.

5. H. Zohm et al., Nucl. Fusion 57, 086002, 2017.

6. O. Dumbrajs, G.S. Nusinovich, IEEE Trans. on Plasma Science, 32, 934-946, 2004.

7. B. Piosczyk et al., IEEE Trans. on Plasma Science, $\underline{25}$, 460-469 (1997).

8. S. Ruess et al., in Proc. EuMW Conference 2017, Nuernberg, Germany, Oct. 2017, EuMC40-5.

9. I. Gr. Pagonakis et al., Physics of Plasmas, 23, 083103 (2016); doi: 10.1063/1.4959113

10. S. Ruess et al., IEEE Transactions on Electron Devices, 63, p. 2104-2109, 2016, 
DOI:10.1109/TED.2016.2540298.

11. I. Pagonakis et al., 20th Joint Workshop on Electron Cyclotron Emission (ECE) and Electron Cyclotron Resonance Heating (ECRH), May 2018, Greifswald, Germany

12. T. Ruess et al., German Microwave Conference (GeMiC 2018), 12 - 14 March 2018, Freiburg, Germany, Conference proceedings (online) DOI: 10.23919/GEMIC.2018.8335085.

13. T. Ruess et al., 20th Joint Workshop on Electron Cyclotron Emission (ECE) and Electron Cyclotron Resonance Heating (ECRH), May 2018, Greifswald, Germany

14. J. Jin et al., IEEE Transactions on Microwave Theory and Techniques, 57, No. 7, pp. 1661-1668, 2009.

15. J. Jin et al., 20th Joint Workshop on Electron Cyclotron Emission (ECE) and Electron Cyclotron Resonance Heating (ECRH), May 2018, Greifswald, Germany

16. K. A. Avramidis et al., IEEE Trans. Electron Devices, January 2018 (online) DOI: 10.1109/TED.2017.2782365.

17. P.C. Kalaria et al., International Vacuum Electronics Conference (IVEC 2018), Monterey, California, 2426 April 2018.

18. L. Jackowski, Advanced Vertical Collector Sweeping for High Power Gyrotrons, Bachelor Thesis, Oct. 2017, KIT, Karlsruhe

19. W. Kdous, Technical Design of an Advanced Vertical Collector Sweeping System for High Power Gyrotrons, Bachelor Thesis, April 2018, KIT, Karlsruhe

20. T. Rzesnicki et al., IEEE Transactions on Plasma Science, VOL. 38, NO. 6, June 2010.

21. T. Rzesnicki et al., 34th International Conference on Infrared, Millimeter, and Terahertz Waves, Busan, 2009, doi: 10.1109/ICIMW.2009.5324919

22. T. Rzesnicki et al, in Proc. 38th IRMMW-THz, Mainz, Germany 2013, We5-3.

23. I. Gr. Pagonakis et al., Physics of Plasma, 23, Page 023105, 2016

24. S. Ruess et al., Vacuum Electronics Conference (IVEC) 2016 IEEE International, pp. 1-2, 2016.

25. J. Zhang et al., Proc. IVEC 2015, Beijing, China, 2015.

26. J. Neilson, Joint 29 th Int. Conf. on IRMMW \& $12^{\text {th }}$ Int. Conf. on Terahertz Elect., Karlsruhe, Germany, 2004, pp. 667-668. 